\title{
Data Privacy Preservation and Security Approaches for Sensitive Data in Big Data
}

\author{
Rohit Ravindra Nikam ${ }^{\mathrm{a}, 1}$, Rekha Shahapurkar ${ }^{\mathrm{b}, 2}$ \\ ${ }^{a}$ Research Scholar,Computer Science and Engineering Department, Oriental \\ University, Indore, India \\ ${ }^{\mathrm{b}}$ Computer Science and Engineering Department, Oriental University, Indore, \\ India
}

\begin{abstract}
Data mining is a technique that explores the necessary data is extracted from large data sets. Privacy protection of data mining is about hiding the sensitive information or identity of breach security or without losing data usability. Sensitive data contains confidential information about individuals, businesses, and governments who must not agree upon before sharing or publishing his privacy data. Conserving data mining privacy has become a critical research area. Various evaluation metrics such as performance in terms of time efficiency, data utility, and degree of complexity or resistance to data mining techniques are used to estimate the privacy preservation of data mining techniques. Social media and smart phones produce tons of data every minute. To decision making, the voluminous data produced from the different sources can be processed and analyzed. But data analytics are vulnerable to breaches of privacy. One of the data analytics frameworks is recommendation systems commonly used by e-commerce sites such as Amazon, Flip Kart to recommend items to customers based on their purchasing habits that lead to characterized. This paper presents various techniques of privacy conservation, such as data anonymization, data randomization, generalization, data permutation, etc. such techniques which existing researchers use. We also analyze the gap between various processes and privacy preservation methods and illustrate how to overcome such issues with new innovative methods. Finally, our research describes the outcome summary of the entire literature.
\end{abstract}

Keywords. Data Privacy, Privacy Preservation Techniques, data mining, anonymization, 1-diversity, bigdata.

\section{Introduction}

Privacy and security are essential issues in big data. A significant data protection paradigm isn't recommended in complicated systems because of which it is deactivated by nature. Nonetheless, details will still be quickly corrupted in the absence of it. As such, this segment focuses on topics of privacy and protection. Protection Records Protection is the luxury of getting any power over the processing and usage of confidential details.

\footnotetext{
${ }^{1}$ Rohit Ravindra Nikam, Research Scholar Computer Science and Engineering Department, Oriental University, Indore; E-mail: rohitnikam3000@gmail.com

2 Dr. Rekha Shahapurkar, Computer Science and Engineering Department, Oriental University, Indore; Email: rekhashahapurkar@orientaluniversity.in
} 
Privacy preservation in data mining is a branch of research that is currently gaining popularity these days. Privacy-preserving is nothing but obtaining data mining algorithm results without compromising the underlying dataset. Data mining algorithms are analyzed whether they are causing any side effects on data privacy. The two-fold is the primary consideration during data mining privacy preservation.

First, all the sensitive raw data in the dataset, which may include contact details, names, addresses, and such demographic details, are identified and changed from the original database. Hence the receiver of this information will not compromise on the authenticity and privacy of other people's personal information. Second, the sensitive information that is being mined as a result of various data mining strategies should also be eliminated since it can also harm an individual's privacy.

The primary focus is on the difficulty of guaranteeing the privacy of information withdrawal results. The meaning of security must fulfil the requirements of clients of a sensible application. Two examples of such application are (1) credit or finance giver, whose clients may comprise of various shops as well as a small business, also who wishes to give them among a classifier that will discriminate which are creditworthy or risky customers, and (2) a medicinal corporation that desires to put out a study identifies the cluster of patients who act in reply another way to a route of action. These information proprietors wish to discharge information mining yield yet be guaranteed that they are not giving the personality of their customers endlessly. Suppose it might be confirmed to free result to withstand limits related to those set by k-anonymity. In that case, the praise donor might free a k-anonymous classifier and dependably declare that the confidentiality of individuals is confined.

The research provides data privacy and security to extensive unstructured data using effective classification and privacy algorithms. The flexibility of the system has measured in a different environment like distributed data systems etc. This work focuses on various existing techniques that used privacy preservation on extensive data with other privacy preservation techniques [1-3].

\section{Existing methodologies}

The Fran Casino et al. [1] illustrate blockchain as the Decentralized RS core, seeking to provide it with a wide variety of functionality while protecting consumer privacy. We are implementing a new design focused on decentralized area sensitive hashing classification and a range of suggestion methods, depending on how users handle data. Extensive study findings show the efficiency and efficacy of our methodology relative to cutting-edge approaches.

Chenyang Ma et al. [2] Describes Separating encrypted transactions in the block and calculating only bilinear pairings on node block cypher texts rather than all ciphertexts, which helps to reduce the computing costs that mining operation. Finally, we test the efficacy of our set of rules by conducting statistical analyses and simulator tests on numerical expenses, safety, precision, and time aspects. The results indicate our protocol would produce the right mining outcome and outperform the prior method in terms of performance under a similar protection standard of conditions. 
Dongfeng Fang et al. [3] explained IoT network architecture, which contains heterogeneous IoT systems listed. Specific confidence models are developed and evaluated in the IoT framework depending on the confidence relationships between the different actors. We suggest a scalable and robust authentication scheme that considers heterogeneous IoT devices based on a paradigm needed for the least trust. The proposed method offers resource-limited IoT users protection and privacy in a scalable and effective way by using IoT users with improved storage and computational ability. Anmin $\mathrm{Fu}$ et al. [4] Tackle this by introducing a novel Non-negative Matrix Factorization (NMF) outsourced scheme that seeks to reduce the computational pressure of consumers and resolve stable issues posed by NMF outsourcing. O-NMF specifically exploits Paillier homomorphism, focused on two non-collusion servers, to protect data privacy. O-NMF provides a testing system to support clients validate returned findings with a high degree of accuracy probability.

Muhammad Usman et al. [5] this system works in three steps. The first level edge devices affect a lightweight aggregation method to produced data during the first step. This approach limits the size of the data produced and seeks to protect data source privacy. In the 2nd tier, a multi-step process used for linking Level Two Edge Devices (LEDs) with High-Level Edge Devices (LEDs). The validation phase-only valid LEDs can move data to the LEDs, resulting in a reduction in the computational burden on LEDs. In the third level, the LEDs use a convolution neural network to predict the position of touching objects in LED data transmitted.

Jiannan Wei et al. [6] Propose a secure, safe and privacy-conserving IoT Message authentication scheme. Our framework embraces IoT devices with varying cryptographic settings and enables offline and online computing, making them more versatile and powerful than previous systems.

Rong Jiang et al. [7] Big Data processing, delivery, analysis, usage and sharing examined protection and privacy leakage possibilities. They recognized a Clinical Big Data Security and Privacy Leakage Possibility Predictor framework amid four leading indicators and thirty minor indicators. Additionally, weight for every variable was determined using the weight system GI and Entropy. The Fuzzy Method of Structured Analysis was recognized to check the principle of Big Data Medical Protection and Urban Privacy Computing.

Karen R. Sollinset. al. [8] explains requirements and limits and proposes a three-part decomposition of architecture. To arrive at this final analysis, we begin by clarifying the issues in the design space:

There is some agreement about what IoT means, particularly on the security and privacy consequences of various definitions.

1. We then consider the requirements and constraints on Big Data resulting from unique IoT system designs.

2. We examine the industry intricacies in parallel.

In this sense, we can then break down the set of drivers and data protection/privacy and innovation goals into1) the history of regularity and social policy; 2) the economic and industrial history; and 3) the context of technology and architecture.

Ismail Hababehet. al. [9] proposes an advanced approach for classifying and safeguarding extensive data while conducting versatility, duplication, and study. The data classification defines the requirement to protect extensive data accessibility into two categories; confidential and public as per the degree of hazard result of information. The effect of data protection is analyzed and authenticated on the subtle data within the framework of the classification group HDFS cluster. 
Si Han et al. [10] proposes a Hidden community exchange key management protocol (SSGK) to avoid unwanted exposure to the contact channel and mutual data. Unlike previous books, the joint data is authenticated with a group key, and a secure distribution mechanism is used to spread the set of keys in SSGK. The detailed protection and efficiency analyses demonstrate that our collection of rules significantly minimizes data contribution's privacy security and hazards in cloud storage and saves on twelve percentage of storage space.

Xiaodan Yan et al. [11] proposed the GAN Model Attackable of deep learning execution; this approach mainly studies the information protection methods under GAN model attack to find a better way to prevent attacks and effectively protect information. Sometimes medical treatment data may be leaked to third-party organizations. When these essential medical data are illegally used by for-profit organizations or obtained by criminals, it will lead to the disclosure of personal privacy information and cause severe economic losses to the victims. However, the victim cannot delete the leaked report by itself or limit the scope and use of the information that has been revealed.

Gamage Dumindu Samaraweera et al. [12] Proposed Protection and Privacy Effects on Big Data Age Database Systems: A study, his paper analyses protection applications in today's essential database models rely primarily on security and privacy attributes. A collection of standard protection measures is defined and tested based on different protection classifications. This offers a thorough overview and study of the sophistication of protection and privacy frameworks in the database models coupled with possible orders/enhancements. Data owners can agree on the maximum appropriate data storage for their data-driven Big data applications.

Yanan Li et al. [13] Propose a data correlation framework effect on privacy leakage, defined as Previous Differential Privacy (PDP), which is recommended to determine information leakage considering the opponent's specific prior awareness. The model uses two methods for evaluating discrete and continuous results, respectively, the weighted hierarchical graph and the multivariate Gaussian model. This further shows the distinct effect of optimistic, negative, and mixed associations on data leakage. A closed structure definition of privacy leak extracted used for unbroken data considering general associations, and a chain law is introduced for separate data.

Yang Liu et al. [14] Preservation of privacy and aggregation through reinforcement learning suggests a Payment-Privacy Protection Level (PPL) game in which each member submit their sensing data along with a given PPL as a system selects the appropriate payment for the participants. In addition to removing the Nash equilibrium (NE) stage of the game. Consider a payment-PPL system is ambiguous; it uses a reinforcement learning strategy, i.e., Q-learning, to get the payment-PPL method in the complicated payment-PPL game. Here it uses a deep Q network (DQN), which combines deep learning and Q-learning to speed up learning.

Gamage Dumindu Samaraweera et al. [15] Proposed defence design review of leading database models of today, with a greater focus on security and privacy attributes. A set of standard protections is specified and assessed based on specific classifications of security. It offers a thorough summary and systematic analysis of the complexity of security and privacy technologies in the database models, together with potential directions/improvements. Data owners may select the most proper data store for their data-driven Big Data technologies models.

David Froelicher et al. [16] proposed Drynx, a Decentralized data management framework Knowledge of mathematical study of distributed databases. The author 
relies on a group of computer nodes to allow data, such as standard deviation or severe, to be computed and to educate and test machine-learning models on critical and distributed data. Drynx incorporates collaborative protocols, holomorphic security, zero information evidence of validity, and differential protection to guarantee user confidentiality and service provider safety. This enables a practical and autonomous inspection of the entry data and all computation of the method, thereby ensuring audit ability in a transparent adversarial environment where no individual is involved has to be independently reliable.

DatThanh Dang et al. [17] proposes a trust-based MapReduce system for activities related to extensive data analysis. Precisely, we are first quantifying and proposing to allocate the critical values for map data and trust values than to minimize slots. Then we measure the faith factor of each tool engaged in the tasks of extensive data analysis. Based on the vulnerability level of a job for the data, the role needs a certain degree of faith (i.e., low important data require higher confidence degree servers/slots). The MapReduce scheduling difficulty is developed for the biggest weighted similar problem of a bipartite graph aimed at optimizing the cumulative confidence factor of all available assignments subject to specific confidence needed tasks.

Guangquan Xu et al. [18] suggested an original and versatile framework named So Protector to avoid privacy leakage by examining data stream linking Java and native layers. So, protector discovers a real-time system that detecting malicious features embedded in SO libraries. Placed, we extract the malware features through 3 steps:

1. Current as a grayscale picture binary files in the native family.

2. Using the ARM instructions package to reverse the SO file code using python to find the op-code sequence.

3. IDA Pro converts all files as an assembly language, which contains a .gdl files as an addition.

Dharminder et al. [19] Introduces a sign encryption strategy focused on identification by adding both encryption and signature that offers an answer to safe and authenticate communication in the Big Data location called SFEEC (Safety Mechanism for EnergyEfficient Computing). By providing pairing-free calculation at the end of the customer, SFEEC fulfils the criteria of fewer overhead computation and connectivity. SEC is also proved in the norm under attacks "indistinguishable from chosen-ciphertext" and "stable against chosen post" model.

Ruiyang Xiao et al. [20] Create a mixing scheme with one shared signature protocol without depending on third parties or having a transaction cost. The method utilizes a bargaining mechanism to maintain transparency of the agreement, supervised by the participants. The system also contains a signature procedure focused on the ElGamal signing procedure and key sharing.

\section{Problem Description}

It might be confirmed so as to free result with stand limits related to those set by kanonymity, after that the praised on or might free a k-anonymous classifier along with dependably declare that the confidentiality of individuals is confined. Similarly, the author of a medicinal study quoting k-anonymous group centroids might be confident that they fulfill with confidentiality principles, which prohibit the make public of independently identifiable physical data. 


\section{Privacy Preservation Techniques}

Privacy preservation in data mining is branch of research that is currently gaining lot of popularity these days. Privacy preserving is nothing but obtaining data mining algorithm results without compromising the underlying dataset. Data mining algorithms are analyzed whether they are causing any side-effects on data privacy.

So, the main motive behind preserving privacy during data mining is to build an approach which modifies the original dataset in such a way that even after performing mining process, the privacy of information remains intact.

K-Anonimity: Slice is fundamentally relying upon rows as well as columns parceling. In multiple attribute dividing, we apportioned information as \{user_name\}, \{user_age, user_zip\}, \{user_disease\} and tuple parceling (even segment) as \{Att[1], $\mathrm{Att}[2] \ldots . . . \mathrm{Att}[\mathrm{n}]\}$. In trait parceling age and zip are apportioned together on the grounds that they both are exceedingly connected in light of the fact that is nothing but a Quasi identifier (QI). The group of QI should be known to assailant. While tuple dividing framework should check L assorted variety of the Sensitive Attribute (SA) fragment [21]. Algorithm has followed the below procedure.

1. First select the specific attribute set from D Att []$\leftarrow \mathrm{D}$ which illustrates the Att set from selected record;

2. While QI not present in record

On the off chance that iSet $\leftarrow$ n

Check L decent variety;

Else

iSet++;

Return Dview *;

3. QSet $=$ QSet $-\left\{\right.$ Dview $^{*}+$ att $\left.[\mathrm{i}]\right\}$;

4. Apply pruning stage 2 and 3 with read up to next tuple in $\mathrm{Q}$

5. Dview*= Dview *UA[Datasize]/ Next Anonimized data from table instance

To begin with describe $\mathrm{k}=$ point of confinement of information anonymization container measure, number of lines, number of sections, exhibit rundown and database in the queue (step 1). Additionally, process will be done if and just if line isn't void i.e there should to be information in database. Check information for L assorted variety if row count $=\mathrm{k}=\mathrm{m}$ (stage 2). At first $\mathrm{Q}=\mathrm{Queue}$ of data. On the off chance that our can information satisfies $\mathrm{k}$ obscurity and L decent variety, it returnsDview* i.e anonymized perspective of information. The database information which can't satisfy prerequisite of protection will put away in exhibit list a [i]. Presently information stays in database i.e in $\mathrm{Q}=\mathrm{Q}-\left\{\mathrm{D}^{*+} \mathrm{a}[\mathrm{i}]\right\}$ (stage 3 ). Rehash stage 2 and stage 3. A[D] is anonymization of information in database. Apply above strides for outstanding information and make new anonymization see which is the association of unique examination and new one i.eDview * = Dview *UA [Datasize].

L diversity: L-Diversity is the process of two shows the number of occurrences of specific attribute in view table. Suppose system supports for $\mathrm{L}=10$ it means it can take max L unique records in single virtual view [22].

Step 1: read each (R from Dataset) 
Step 2: SpliteDataParts[] $\leftarrow$ R.split ()

Step 3: check TableViewLcount $=$ L-Diversity count

Step 4: CurrentValue $=$ R.get(Lvalue)

Step 5: If (CurrentValue.notexist (TableView))

TableView.add $\leftarrow$ CurrentValue

Step 6: else

Go to step 1;

Step 7: If (TableView.count> L)

Early stop

Flag=1;

Step 8: continue

Step 9: if (flag==1)

RetrunTableView

Basically 1-diversity illustrates the number of attribute of lenses in specific view. L-Diversity can be holding the privacy scenario during the data distribution. In secure multiparty computation protocol preferred the and privacy as well as hell diversity. In the proposed work system and illustrates the features of L-Diversity. e.g., Algorithm set the specific value for a liability of lenses in particular view in the first time of algorithm system read each row from data table and then data table has a split into the different column values. The desired column considered as a cause identifier for LDiversity and then distributes the data in network environments. The L-Diversity upper limit checks the condition in algorithm in step 8. L-Diversity sometimes communicates with K- anonymity. Both combinations should be forcing the information breaching issue as well as data leakage.

Random Permutation: The permutation is the process which shuffles data attributes as well as privacy attribute randomly. This algorithm first decides the virtual fuel set value according to data type as well as number of rows. During the data distribution system Shuffle some sensitive information at specific attribute to another instances. The benefit of such random permutation approach, it generates half knowledge two traitors or attacker, which provides security from third party attacks like man-in-themiddle etc [23].

F-score for fitness: Basically F-Score is the function that evaluate the current Virtual Table view fitness spelling with normalize attributes. F-Score works with k anonymity as well as 1 diversity. Both the values were works with combine approach which generates the run time fitness score based on the occurrences [24].

Constraint C: Basically C-Constraint is the protocol which verify the security you which is generated by privacy approach. The two-approach carried out to achieve the privacy during the data distribution, first slicing and second randomization as well as generalization. C-Constraint is the policy which works for how much data we can publish in a single view. Basically, it works according to column and rows values. The column values called as L-diversity and rows values called as $\mathrm{k}$ - anonymity, CConstraints evaluate policy when distribution mechanism add the data in virtual view. Below is the process to evaluate heat $\mathrm{C}$-Constraint during the data distribution

1. Define the K-anonimity as well as L-Diversity upper limit by system

2. Define the privacy constraint minimum score fitness score $=1$; 


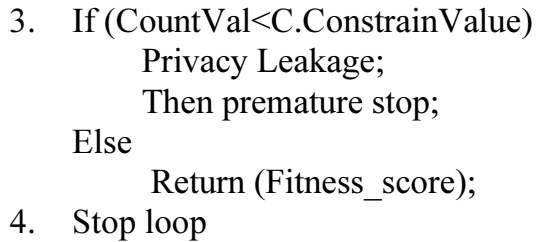

Verification for quality of framework against number of suppliers: For check against number of suppliers, include one more property in anonymized information as a supplier to yield. This confirmation will demonstrate that our procedure of anonymization doesn't rely upon number of suppliers. Existing framework i.e supplier mindful anonymization calculation relies upon database and additionally supplier.

1. Create value of SA by means of Data Generator $P=1 \ldots . . n$

2. ensure for confidentiality constraint also Fscore=1 through number of provider

3. If

Privacy Leack;

next premature stop;

Else

Return (Fitness score);

4. Exit

Consider a potential assault on shared information distributing. We utilized cutting calculation for anonymization and $\mathrm{L}$ assorted variety and confirm it for security and protection by utilizing double calculation of information security. Cutting calculation is extremely valuable when we are utilizing high dimensional information. It partitions information in both vertical and flat mode. Because of encryption we can expand security.Be that as it may, the restriction is there could be loss of information utility. Above framework can utilized as a part of numerous applications like clinic administration framework, numerous modern zones where we get a kick out of the chance to ensure delicate information like compensation of representative. Pharmaceutical organization where touchy information might be a mix of elements of solutions, in saving money segment where delicate information is account number of clients, our framework can utilize.It can be utilized as a part of military region where information is accumulated from various sources and need to anchor that information from each other to look after security.This projected framework helps to enhance the information protection and security when information is assembled from various sources and yield ought to be in cooperative mode.

We first formally depict our concern setting. At that point, we display our information protection definition as for a security imperative to forestall surmising assaults by information enemy, trailed by properties of this new security idea. Let $\mathrm{T}=$ $\{\mathrm{t} 1, \mathrm{t} 2, \ldots\}$ be an arrangement of records with similar qualities accumulated from $\mathrm{n}$ information suppliers $\mathrm{P}=\{\mathrm{P} 1, \mathrm{P} 2, \ldots, \mathrm{Pn}\}$, with the end goal that $\mathrm{Ti}$ are records given by Pi. Let AS be a delicate property with an area DS. In the event that the records contain various delicate traits at that point, we regard every one of them as the sole touchy property, while staying ones we incorporate to the semi-identifier. Nonetheless, for our situations we utilize an approach, which saves greater utility without giving up protection. 
The objective is to distribute an anonymized $\mathrm{T}^{*}$ while keeping any information enemy from surmising AS for any single record.An information foe is a coalition of information clients with $\mathrm{n}$ information suppliers participating to rupture protection of anonymized records. At the point when information is accumulated and joined from various information suppliers, primarily two things are done, for anonymization process. To shield information from outer beneficiaries with certain foundation learning $\mathrm{BK}$, I expect a given protection prerequisite $\mathrm{C}$ is characterized as a conjunction of security imperatives: $\mathrm{C} 1, \mathrm{C} 2 \ldots \mathrm{Cn}$.

If a gathering of anonymized records $\mathrm{T}^{*}$ fulfils $\mathrm{C}$, we say $\mathrm{C}\left(\mathrm{T}^{*}\right)=$ genuine. By definition $C(\varnothing)$ is valid and $\varnothing$ is private. Any of the current protection standards can be utilized as a part requirement $\mathrm{Ci}$.We presently formally characterize an idea of information security regarding a protection requirement $\mathrm{C}$, to ensure the anonymized information against information enemies. The thought expressly models the intrinsic information learning of an information foe, the information records they together contribute, and requires that every QI gathering, barring any of those records possessed by an information foe, still fulfils C. It also demonstrates our security framework in which input information is given from various suppliers. Select point for cutting. Watch that information against security imperative $\mathrm{C}$ for information protection. Check additionally is cutting is conceivable or not. On the off chance that cutting conceivable at that point do it and if not then show the yield information. Our last yield $\mathrm{T}^{*}$ are anonymzed information which will see just by validate client. Any for cannot break security of information. In this framework we are utilizing level and also vertical parcelling over database. Cutting calculation gives better segment parcelling. To comprehend this legitimately we should consider doctor's facility administration framework for explore. Let diverse offices are the suppliers who give information from various sources. We think about ailment as an AS (delicate property) and age and zip code are QI (semi-identifier) [25].

\section{More Privacy approach}

(i) Explicit Identifiers is an arranged of properties containing data that perceives a record director unequivocally, for instance, name, rate et cetera.

(ii) Quasi Identifiers is an arranged of properties that could possibly perceive a record administrator when joined with freely accessible information.

(iii) Sensitive Attributes is an arranged of properties that contains tricky individual specific data, for instance, sickness, pay et cetera.

(iv) Non-Sensitive Characteristics is an organized of properties that makes no issue if revealed even to plotting social events. Information anonymization empowers the exchange of data over a limit, for instance, between two offices inside an organization or between two offices, while diminishing the danger of unintended exposure, and in specific conditions in a way that empowers assessment and investigation post anonymization. With regards to medicinal information, anonymized information insinuates information from which the patient can't be recognized by the beneficiary of the data. The name, address, and full post code must be evacuated together with whatever other data which, in conjunction with other information held by or unveiled to the beneficiary, could distinguish the patient. 


\section{The bottom-up algorithm -}

The base up algorithm is like the Top-down algorithm. The principal contrast is in the succession of coalition checks, which is in a base up form beginning from 0 foe, and moving up. The algorithm stops if an infringement by any foe is distinguished (early stop) or all $\mathrm{m}$-foes are checked.

Algorithm displays the general thought of bottom-up speculation strategy. It starts the speculation from the crude information table $\mathrm{T}$. At every emphasis, the algorithm voraciously chooses the best speculation $g$ that limits the data misfortune and augments the protection pick up. This instinct is caught by the data metric ILPG $(\mathrm{g})=\operatorname{IL}(\mathrm{g}) / \mathrm{PG}(\mathrm{g})$. At that point, the algorithm plays out the speculation child (Best) $\rightarrow$ Best on the table T, and rehashes the cycle until the point that the table $\mathrm{T}$ fulfills the given k-anonymity necessity.

Bottom-Up Generalization: Bottom-Up Generalization is a proficient k-anonymity technique. In a k-mysterious informational collection, each record is indistinct from at any rate k-1 different records concerning QID. Fundamentally, Bottom-Up Generalization (BUG) approach of anonymization is an iterative procedure beginning from the most reduced anonymization level. We use the data/security exchange off as the scan metric for our approach, i.e., the Information Loss per Privacy Gain (ILPG). The Advanced BUG comprises of following advances, information segment, run the MRBUG Driver on apportioned informational index, joining the anonymization levels of the parceled informational collection and applying speculation to unique informational index with incorporated anonymization level without abusing the kanonymity [21-22].

\section{Algorithm Bottom-Up Generalization}

1: while T NOT (assure a given k-anonymity necessity)

perform

2: intended for all simplification $\mathrm{g}$ do

3: calculate ILPG(g);

4: end for

5: get the Best generalization;

6: generalize $\mathrm{T}$ by Best;

7: end while

8: output T;

Let $\mathrm{A}(\mathrm{QID})$ and $\mathrm{Ag}(\mathrm{QID})$ be the base anonymity tallies in $\mathrm{T}$ when the speculation g. Given an information table $\mathrm{T}$, there are numerous conceivable speculations that can be performed. However, most speculations $g$ in reality does not influence the base anonymity tally. As it were, $\mathrm{A}(\mathrm{QID})=\mathrm{Ag}(\mathrm{QID})$. In this manner, to encourage productively picking a speculation $\mathrm{g}$, there is no compelling reason to think about all speculations. To be sure, we can concentrate just on the "basic speculations" [24].

\section{The Top-Down Specialization (TDS) Privacy Approach}

The Top-down algorithm checks the coalitions in a best down manner utilizing descending pruning, beginning from (n-1)-adversaries, and moving down until the point when an infringement by a m-adversary is distinguished or all $\mathrm{G}$ m-foes are pruned or checked.

In this technique we break down the adaptability issue of existing TDS approaches when we taking care of huge scale informational indexes on HADOOP platform. TDS is rehashed process which is beginning from the highest space esteems in the course of action trees of traits. Finding the best specialization, performing specialization and 
updating estimations of the hunt metric. Such a procedure of TDS is rehashed until the point that k-anonymity is damaged, to depiction for the greatest information will use in that. The exemplary nature of a specialization is estimated by an inquiry metric the distinctive android application authorization is brought from android applications. These authorizations are utilized as dataset for process. In that we acknowledge the data pick up per protection misfortune (IGPL), a tradeoff metric that take as a primary concern both the security and data necessities, as the inquiry metric in our approach. A specialization with the most elevated IGPL esteem is viewed as best one and chose of each round. Whenever answer for Top-Down Specialization User may advance through every specialization to decide a coveted exchange off amongst protection and precision. Client may stop whenever and acquire a summed-up table fulfilling the anonymity necessity. Taking care of both downright and ceaseless traits. Progressively produce scientific categorization tree for constant qualities.

A few miniaturized scale information anonymization methods have been proposed. The most mainstream ones are speculation for k-anonymity and bucketization for '1decent variety. In the two methodologies, properties are parceled into three classifications:

1) A few properties are qualifier that can exceptionally recognize personnel, for example, Name or Social Security Digit;

2) A few properties are Quasi Identifiers (QI), that the enemy may definitely know (conceivably from other freely accessible repositories) and which, once considered together, can possibly recognize personnel, e.g., Birth date, Sex, and Zip code;

3) A few characteristics are Sensitive Attributes (SAs), that obscure to the foe and are viewed as touchy, for example, Disease and Salary.

Among speculation and bucketization, one initially expels identifiers from the information and after that segment's tuples into cans. The two methods contrast in the following stage. Speculation changes the QI-values in each container into "less particular yet semantically reliable" qualities with the goal that tuples in a similar can can't be recognized by their QI esteems. In bucketization, one isolates the SAs from the QIs by arbitrarily permuting the SA esteems in each container. The anonymized information comprises of an arrangement of pails with permuted delicate property estimations.

Cutting segments, the informational collection both vertically and on a level plane. Vertical parceling is finished by grouping qualities into segments in view of the relationships among the properties. Every section contains a subset of properties that are profoundly related. Even dividing is finished by grouping tuples into horizontal partitioning. At long last, inside each can, values in every section are haphazardly permutated (or arranged) to break the connecting between various segments. The essential thought of cutting is to break the affiliation cross sections, yet to safeguard the relationship inside every segment. This decreases the dimensionality of the information and jam preferred utility over speculation and bucketization. Cutting jam utility since it groups exceptionally associated properties together, and conserve the relationships between such traits. Slicing ensures protection as this disrupts the relationship among non-related traits, which are rare and consequently distinguishing. Consider that while the informational index comprises QIs and one SA, bucketization needs for disrupting connection; cutting, then again, can merge few QI qualities along through SA, protecting property relationships per delicate characteristic. The primary instinct that cutting gives security assurance is that the slicing procedure guarantees that for any tuple, there are for the most part numerous coordinating containers [22] [23]. 


\section{Proposed system design}

The privacy techniques follow various security techniques like slicing, anonymity, generalization, permutation, etc. When the system deals with a large amount of data, it works with a slicing approach where attributes are suppressed or general awaiting every line is the same through at slightest k-1 extra rows. At this point, the record is supposed to be k-anonymous. It can distinguish between access and non-access details of the Data set. The slicing approach is included in the column separation method. Anonymization of data is yet another approach that eliminates confidential Details to guard user privacy. It is also known as to de-identify. Whenever organizations disclose the data is publicly anonymized. The ideas of k-anonymity, l-diversity, and t-closeness have been used to keep information from re-identification presented.

\section{Privacy Threats in Analytics}

Privacy is a person's ability to determine what data can be shared and to use privileged access. If the data is in the public interest, it constitutes a threat to the user's privacy because the data holder owns the information. Data holders can be served for social networking, blogs, mobile phones, e-commerce platform, banks, hospitals, etc. The data holder is responsible for maintaining the protection of consumer data. Besides the data held in the public domain, directly or indirectly, users are contributing to data leakage. For example, most mobile apps seek access to our contacts, flesh, camera, etc., and we agree to all terms of service without reading the privacy policy by leading to the leakage of data. Therefore, there is a need to inform users of smartphones about risks to safety and privacy. Many of the main dangers to privacy include (1) surveillance; (2) Disclosure; (3) discrimination; (4) Personal embracement or abuse.

\section{Surveillance}

Many organizations, including retail, e-commerce, etc., study their customer's buying habits and try to develop various offers and value-added services [4]. Based on the opinion data and sentiment analysis, social media sites provide recommendations for new friends, places to be seen, people to be observed, etc. This is possible only when they continuously monitor their customer's transactions. This is a severe privacy threat as no Individuals accept oversight.

\section{Disclosure}

Find a hospital keeping data that includes (Zip, gender, age, illness) [5-7]. The data holder has released data for analysis to a third party through anonymization of sensitive data Person shall specify data so as not to identify the person. The third-party data analyst can map this information with the publicly accessible external data sources, including census data, and place a person with a particular disorder. This is how the private data of A person who is deemed to be a severe breach of privacy may be disclosed.

\section{Discrimination}

Discrimination is the bias or injustice that may arise when any person's private information is revealed. Statistical analysis of election outcomes, for example, has proved itself that people of one community were entirely against the governmentforming party. Now the government may ignore, or discriminate towards, the culture. 


\section{Personalized embrace and abuse}

This can also lead to intimate embrace or harassment if any person's private information is revealed. A guy, for example, was undergoing treatment privately for some particular problem and regularly bought some medicines from the doctor's shop. The medical shop can submit some of them as part of its daily business model Reminder and offers relating to these medicinal products by the cell when one member of the family has Seen that would result in the personal embrace and even abuse [8].

Activity in data analytics will affect data privacy. In many countries, privacy is enforced on the Lawson Preservation. Lack of awareness is also a big reason for the attacks on privacy. Because Example: Many smartphone users do not know the information stolen from Multiple apps to their phones. Previous research shows that only $17 \%$ of Mobile users are Aware of the privacy risks [9].

Few basic things have considered by various privacy techniques during data broadcasting without any existing security approach.

1: Key attributes: Based on the attributes uniquely identifies tuples. Ex: Social security number, pan number, adhar number, voter id, driving license number, etc.

2: Quasi Identifiers: An arrangement of traits that can be conceivably connected with outside data to re-distinguish entities. Ex: Zipcode, date of birth, sex.

3: Sensitive attributes: Some of the features contain sensitive value concerning the data owner. Ex: salary and disease.

4: Non-sensitive attribute (NSA): Disclosing the non-sensitive attributes will not break the secrecy of the user.

K-anonymity: It can be used to prevent record linkage. To preserve privacy, the following Anonymization techniques are applied to the data $[9,10,11]$.

Suppression: Quasi-identifiers is supplanted or darkened by some steady qualities like 0 , *, etc. Ex: some values license number, aadhar number can be invisible using an asterisk.

Generalization: Some values are replaced by parent values [3] [4].

The above techniques used to achieve privacy on heterogeneous datasets when data has broadcast in a vulnerable environment.

\section{Results and Discussion}

As part of a systematic analysis of literature, it was found that all current privacy protection measures are about structured data. About $80 \%$ of the data is unstructured that it is produced today. As such, the following needs to be discussed Challenges ahead. Create practical approaches to protect privacy in structured and unstructured ways. Scalable and reliable techniques to be built for heterogeneous handling of large scales datasets. The data should be allowed to remain in its native form without transformation, and data analytics can be carried out while safeguarding privacy. Advanced innovations must be developed aside from anonymization to ensure protection against critical threats to privacy, including disclosure of identity, discrimination, surveillance, etc. Optimizing the value of data while maintaining the confidentiality. In below table we demonstrated an evaluation of system with numerous existing systems. 
Table 1: Comparative analysis between proposed V/S existing approaches with different supplied data size

\begin{tabular}{|l|c|c|l|l|}
\hline \multicolumn{1}{|c|}{ Method } & $\mathbf{1 0 0} \mathbf{k b}$ & $\begin{array}{c}\mathbf{2 0 0} \\
\mathbf{k b}\end{array}$ & $\mathbf{3 0 0 k b}$ & $\begin{array}{c}\mathbf{5 0 0} \\
\mathbf{k b}\end{array}$ \\
\hline $\begin{array}{l}\text { Privacy approach using top-down } \\
\text { generalization }\end{array}$ & 246 & 488 & 723 & 975 \\
\hline $\begin{array}{l}\text { Enhanced Slicing Models for Preserving } \\
\text { Privacy in Data Publication }\end{array}$ & 310 & 602 & 923 & 1178 \\
\hline $\begin{array}{l}\text { Privacy using Anonimization approach } \\
\begin{array}{l}\text { privacy protection and fingerprint generation } \\
\text { (Proposed) }\end{array}\end{array}$ & 235 & 400 & 650 & 800 \\
\hline
\end{tabular}

The above Table 1 depicts the time performance to generate the privacy view of proposed system with some existing approaches. The proposed approach is improving the time around $5 \%$ over all existing approaches.

\section{Conclusion}

We have shown in this article that various techniques and methods of privacy preservation, i.e., k-anonymity, l-diversity, t-closeness, provide strong privacy in big data. No practical approach has yet been built on unstructured data. Standard classification and clustering problems can be applied with data mining algorithms but can't protect privacy, mainly when dealing with specific individual information. It's information. It could be used to improve machine learning and soft computing techniques, new and more acceptable solutions to privacy issues, including disclosure of identities that may lead to personal awkwardness and abuse. There are several ways of working in the future. To protect privacy in the future, when interests of the data and data variety enhance, novel analysis is applied.

\section{Acknowledgement}

I would like to express my deep gratitude to Research Dean, Head of Department of Computer Science and Engineering, my research supervisor valuable guidance for providing required resources to carry out research work. I would also like to thank Honorable Managing Trustee of Sanjivani College of Engineering, Kopargaon, India, Head of Department of Information Technology for providing support for the research work.

\section{References}

[1]. Casino F, Patsakis C. An Efficient Blockchain-Based Privacy-Preserving Collaborative Filtering Architecture.IEEE Transactions on Engineering Management.2019 Oct 22. 
[2]. Ma C, Wang B, Jooste K, Zhang Z, Ping Y. Practical Privacy-Preserving Frequent Itemset Mining on Supermarket Transactions. IEEE Systems Journal.2019 Jun 26.

[3]. Fang D, Qian Y, Hu RQ. A Flexible and Efficient Authentication and Secure Data Transmission Scheme for IoT Applications, IEEE Internet of Things Journal.2020 Feb 3.

[4]. Fu A, Chen Z, Mu Y, Susilo W, Sun Y, Wu J. Cloud-based Outsourcing for Enabling Privacy-Preserving Large-scale Non-Negative Matrix Factorization. IEEE Transactions on Services Computing.2019 Aug 28.

[5]. Usman M, Jolfaei A, Jan MA. RaSEC: An Intelligent Framework for Reliable and Secure Multi-Level Edge Computing in Industrial Environments. IEEE Transactions on Industry Applications.2020 Feb 20.

[6]. Wei J, Phuong TV, Yang G. An Efficient Privacy-Preserving Message Authentication Scheme for Internet-of-Things.IEEE Transactions on Industrial Informatics.2020 Feb 10.

[7]. Jiang R, Shi M, Zhou W. A Privacy Security Risk Analysis Method for Medical Big Data in Urban Computing. IEEE Access. 2019 Sep 24; 7:143841-54.

[8]. Sollins KR. IoT big data security and privacy versus innovation. IEEE Internet of Things Journal. 2019 Feb 15;6(2):1628-35.

[9]. Hababeh I, Gharaibeh A, Nofal S, Khalil I. An integrated methodology for big data classification and security for improving cloud systems data mobility. IEEE Access. 2018 Dec 28; 7:9153-63.

[10]. Han S, Han K, Zhang S. A Data Sharing Protocol to Minimize Security and Privacy Risks of Cloud Storage in Big Data Era. IEEE Access. 2019 May 3; 7:60290

[11]. Shuo Zhang, Yaping Liu, Shudong Li, Zhiyuan Tan, Xiaomeng Zhao, Junjie Zhou, "FIMPA: A Fixed Identity Mapping Prediction Algorithm in Edge Computing Environment", Access IEEE, vol. 8, pp. 17356-17365, 2020.

[12]. Samaraweera GD, Chang MJ. Security and Privacy Implications on Database Systems in Big Data Era: A Survey. IEEE Transactions on Knowledge and Data Engineering.2019 Jul 18.

[13]. Y. Li, X. Ren, S. Yang and X. Yang, "Impact of Prior Knowledge, and Data Correlation on Privacy Leakage: A Unified Analysis," in IEEE Transactions on Information Forensics and Security, vol. 14, no. 9, pp. 2342-2357, Sept. 2019.

[14]. Liu Y, Wang H, Peng M, Guan J, Xu J, Wang Y. DeePGA: A Privacy-Preserving Data Aggregation Game in Crowdsensing via Deep Reinforcement Learning. IEEE Internet of Things Journal.2019 Dec 3.

[15]. Samaraweera GD, Chang MJ. Security and Privacy Implications on Database Systems in Big Data Era: A Survey. IEEE Transactions on Knowledge and Data Engineering.2019 Jul 18.

[16]. Froelicher D, Troncoso-Pastoriza JR, Sousa JS, Hubaux JP. Drynx: Decentralized, Secure, Verifiable System for Statistical Queries, and Machine Learning on Distributed Datasets. arXiv preprint arXiv:1902.03785. 2019 Feb 11.

[17]. Dang DT, Hoang D, Nguyen D. Trust-based Scheduling Framework for Big Data Processing with MapReduce. IEEE Transactions on Services Computing.2019 Sep 3.

[18]. Potharaju, S. P. (2018). An Unsupervised Approach For Selection of Candidate Feature Set Using Filter Based Techniques. Gazi University Journal of Science, 31(3), 789-799.

[19]. D. Dharminder, M. S. Obaidat, D. Mishra, and A. K. Das, "SFEEC: Provably Secure Signcryption-Based Big Data Security Framework for Energy-Efficient Computing Environment," in IEEE Systems Journal March 2020.

[20]. Potharaju, S. P., \& Sreedevi, M. (2018). A novel cluster of quarter feature selection based on symmetrical uncertainty. Gazi University Journal of Science, 31(2), 456-470.

[21]. Arava K, Lingamgunta S. Adaptive k-anonymity approach for privacy preserving in cloud. Arabian Journal for Science and Engineering. 2019 Jul 16:1-8.

[22]. Yao L, Chen Z, Hu H, Wu G, Wu B. Sensitive attribute privacy preservation of trajectory data publishing based on 1-diversity. Distributed and Parallel Databases. 2020 Nov 17:1-27.

[23]. Chen J, Liu G, Liu Y. Lightweight privacy-preserving raw data publishing scheme. IEEE Transactions on Emerging Topics in Computing. 2020 Feb 17.

[24]. Yin C, Shi L, Sun R, Wang J. Improved collaborative filtering recommendation algorithm based on differential privacy protection. The Journal of Supercomputing. 2020 Jul;76(7):5161-74.

[25]. Batmaz Z, Kaleli C. Methods of privacy preserving in collaborative filtering. In 2017 International Conference on Computer Science and Engineering (UBMK) 2017 Oct 5 (pp. 261266). IEEE. 